\title{
Research on Institutional Affirmation Factor Based on Structural Equation Model
}

\author{
Fan Jiangyan, Zhang Hongbing \\ Basic Department, Engineering University of PAP, Xi'an, China \\ Email address: \\ 654396497@qq.com (Fan Jiangyan), 100272347@qq.com (Zhang Hongbing)
}

\section{To cite this article:}

Fan Jiangyan, Zhang Hongbing. Research on Institutional Affirmation Factor Based on Structural Equation Model. Science Discovery. Vol. 7, No. 6, 2019, pp. 453-457. doi: 10.11648/j.sd.20190706.23

Received: November 25, 2019; Accepted: January 3; Published: January 4, 2020

\begin{abstract}
The affirmation of the country's existing system by the nationals is the basic element and an important manifestation of national security. As an important force for safeguarding national security and social stability, the military's national defense education is far different from the locality in terms of breadth and depth, and its functional mission makes the group have higher political awareness requirements. Therefore, the hypothesis of this paper is that the affirmative factor of the military group is significantly better than that of the non-military group. By selecting samples of military personnel or former military personnel in China's Comprehensive Social Survey (CGSS), and randomly extracting the same number of non-military samples, a structural equation model was constructed to analyze the differences and internal mechanisms. The results show that this hypothesis is established, and there is a strong correlation between government satisfaction factors and institutional affirmative factors. Life satisfaction, social attitudes and political attitude factors also affect system security to some extent.
\end{abstract}

Keywords: Institutional Affirmation Factor, Military, Difference Analysis, Intrinsic Mechanism, Structural Equation Model, Institutional Security

\section{基于结构方程模型的制度肯定性因子研究}

\section{樊江燕, 张红兵}

武警工程大学基础部, 西安, 中国

\section{邮箱}

654396497@qq.com（樊江燕）, 100272347@qq.com（张红兵）

摘要: 国民对我国现有制度的肯定是国家安全的基础要素和重要体现。军队作为维护国家安全和社会稳定的重要 力量, 其接受的国防教育从广度到深度都远不同于地方, 且其担负的职能使命使得该群体有较高的政治意识要求。 因此本文作出假设：军人群体的肯定性因子要显著好于非军人群体。通过选取中国综合社会调查（CGSS）中军人 或者曾经是军人的样本, 并随机抽取出等量的非军人样本, 构建结构方程模型进行差异性以及内在作用机制分析。 结果表明这一假设是成立的, 且政府满意度因子与制度肯定性因子间存在较强关联, 生活满意度、社会态度以及 政治态度因子也在一定程度上影响着制度安全。

关键词: 制度肯定性因子, 军人, 差异性分析, 内在作用机制, 结构方程模型, 制度安全 


\section{1. 引言}

美国心理学家马斯洛1943年提出需求层次理论, 将安 全视为人在满足生理需求后的第一需求, 并指出整个有机 体就是一个追求安全的机制。“国家安全”这一概念是何时 出现的? 基于英国学者曼戈尔德 (Peter Mangold) 的考证, “国家安全”一词的用法最早是在美国报纸专栏作家李普 曼1943年的著作《美国外交政策》中[1]。国家是否安全, 是每一个主权国家首要关注的问题, 其构成要素随着历史 的演变以及国际国内形势的变化, 也在不断的调整和发展。 我国国家安全要素从毛泽东时代的“军事安全、国土安全、 政治安全”, 经过邓小平时代、江泽民时代以及胡锦涛时 代的演变, 到习近平时代国家安全已发展为包含十一个要 素的结构体系[2]。在这一安全体系中, 政治安全是根本与 保障[3]。有学者指出, 在保障政治安全的四大因素——强 制力、政绩、制度和意识形态中，制度因素起根本性支撑 作用[4]。

当下，我国正处于从大国迈向强国的关键阶段，“树 大招风”效应使得国内外对我国的渗透与破坏行动日益加 剧[5]。在政治安全上, 西方势力企图发动“颜色革命”; 在 国土安全上, “台独”“港独”分裂势力步步紧逼; 在经济安 全上, 中美贸易摩擦给我国的经济稳定带来影响.....安全 形势不容乐观[6]。如何维护好我国内部的安全稳定, 抵御 意识形态领域的冲击与侵蚀, 是打赢这场战争的关键。制 度安全作为国家安全的基础性和根本性要素, 我们必须牢 牢稳固其根基。

作为有着较高政治意识要求群体的军人[7], 他们在制 度安全上的表现是否好于其他群体? “全民皆兵”的理念 是否可以在当代意识形态领域付诸实践 [8]? 影响人们制 度认可程度的因素有哪些? 本文基于中国综合社会调查 (CGSS) 数据库, 以结构方程模型[9]为工具, 对这些问 题作以初步的思考并提供数据上的支撑。

\section{2. 研究基础}

\section{1. 方法介绍}

结构方程模型[9]可用于解决对复杂多变量研究数据 的探究与分析。本文利用结构方程模型中的探索性因素分 析[10]与验证性因素分析[11]对公民关于我国现有制度的 认可情况展开研究。探索性因素分析与验证性因素分析都 是以普通因子分析模型作为理论基础, 是利用少数几个因 子来反映整体多个随机变量间交错复杂关系的一种方法, 通过对因子的提取, 完成原始变量的分类, 实现降维 [12]。 提取出的公因子可以对研究对象进行更好的解释, 并实现 对制度肯定性因子的客观评价。

\section{2. 数据来源}

本文的研究数据来源于中国综合社会调查 (CGSS), 采用最新开放的CGSS2015年度调查数据进行分析。2015 年CGSS项目调查覆盖全国28个省/市/自治区的478个村居, 共完成有效问卷10968份。由于本文研究对象的相对特殊 性以及在总人口中所占比例较少, 根据以下四个题目“您
目前的户口登记状况” (军籍)、“您获得非农户口的途径” (参军)、“您目前工作的单位或公司的单位类型” (军队)、 “您最近那份非农工作的单位或公司的单位类型”(军队), 创建新变量“是否进入过军队体制”, 節选出有过部队经历 或当下是现役身份的样本共 105 份。为避免在单因素方差 分析中样本量失衡带来分析误差, 本文在剩余总体中通过 SPSS软件随机抽取 1\%作为对照组, 最终研究样本总量为 211。

合理、有效的选取评价指标是准确研究制度肯定性因 子的关键。为确保全面, 本文基于社会态度、生活满意度、 政治态度和政府满意度四个层面从CGSS（2015）问卷中 提取出 12 个与制度肯定性因子可能存在相关性的问题, 构 建起本文研究的指标体系（表1）。

表1 对现有制度的肯定性因子评价指标。

\begin{tabular}{ll}
\hline 指标 & 变量 \\
\hline 您同不同意在这个社会上绝大多数人都是可信任的 & $\mathrm{X} 1$ \\
您认为当今社会公不公平 & $\mathrm{X} 2$ \\
在不直接涉及金钱利益的一般社会交往中, 您觉得下列人士 & $\mathrm{X} 3$ \\
可以信任的人多吗 & $\mathrm{X} 4$ \\
您觉得您的生活是否幸福 & $\mathrm{X} 5$ \\
与同龄人相比, 您认为您的社会经济地位如何 & $\mathrm{X} 6$ \\
您认为您目前的收入是否合理 & $\mathrm{X} 7$ \\
如果有人在公开场所发布批评政府的言论, 政府不应干涉 & $\mathrm{X} 8$ \\
生多少孩子是个人的事, 政府不应该干涉 & $\mathrm{X} 9$ \\
在哪里工作和生活是个人的自由, 政府不应该干涉 & $\mathrm{X} 10$ \\
您对政府在下列工作方面的表现如何 & $\mathrm{X} 11$ \\
您对政府所提供的以下公共服务的满意度如何 & $\mathrm{X} 12$ \\
您对我国目前公共服务总体上的满意度如何 & \\
\hline
\end{tabular}

\section{3. 研究假设}

假设一: 进入过军队体制的群体对现有制度的认可程 度显著高于其他人。

假设二：生活满意度越高，社会态度表现越正向。

假设三: 生活满意度越高、社会态度越正向, 对政府 的满意度越高。

假设四: 生活满意度与社会态度会通过政府满意度的 中介作用，间接影响政治态度。

\section{3. 模型构建}

\section{1. 探索性因素分析}

\subsection{1. 数据检验}

为判断构建的指标体系是否符合因子分析的要求, 本 文通过 $\mathrm{KMO}$ 检验以及巴特利特球形度检验进行适配性分 析。经SPSS分析得到KMO值为 0.700 , 巴特利特球形度检 验结果显著, 因此可以进行因子分析。

表2 $\mathrm{KMO}$ 和巴特利特球形度检验结果。

\begin{tabular}{lll}
\hline KMO 取样适切性量数。 & & $\mathbf{0 . 7 0 0}$ \\
\hline \multirow{2}{*}{ 巴特利特球形度检验 } & 近似卡方 & 329.141 \\
& 自由度 & 66 \\
& 显著性 & 0.000 \\
\hline
\end{tabular}




\subsection{2. 公因子提取及解释说明}

本文按照特征值大于 1 的标准提取公因子[13]。如表3 所示, 前 4 个公因子的特征值大于 1 , 因此将从 12 个指标变 量中提取 4 个公因子, 累计解释原始指标变量总方差的 $55.272 \%$ 。其中第一个公因子方差占总方差的 $15.374 \%$, 解 释能力最强。在旋转后的成分矩阵中（表4）, 第一个因 子在公共服务资源的充足程度等四个方面的总体评价 (X12)、政府打击犯罪等九个工作方面的表现(X10)以及 政府所提供的医疗卫生等公共服务的满意度(X11)上载荷 较高, 主要反映群众对政府工作的认可程度, 因此命名其 为政府满意度因子 $(\mathrm{GOV})$; 第二个公因子在自我社会经
济地位感知(X5)、收入满意度 $(\mathrm{X} 6)$ 以及生活幸福感知 $(\mathrm{X} 4)$ 上具有较高的载荷, 反映人们对自己当下生活状况的满意 程度, 将其命名为生活满意度因子(LIF)；第三个公因子 在对社会上绝大多数人的信任感评价 (X1)、不涉及金钱 利益的社会交往中信任感评价 (X3) 以及对社会是否公平 的感知 (X2) 上具有较高载荷, 反映了人们的社会信任程 度, 命名其为社会态度因子 $(\mathrm{SOC})$; 第四个公因子在政 府是否应该干预个人的工作和生活地点 (X9) 、政府是否 应该干预个人生孩子的多少 (X8)、如果有人在公共场所 发布批评政府的言论政府是否应该干涉 (X7) 上具有较高 载荷, 命名其为政治态度因子 (POL)。

表3 总方差解释。

\begin{tabular}{|c|c|c|c|c|c|c|}
\hline \multirow{2}{*}{ 主成分 } & 初始因子解 & \multicolumn{5}{|c|}{ 旋转载荷平方和 } \\
\hline & 总计 & 方差百分比 & 累积 $\%$ & 总计 & 方差百分比 & 累积 $\%$ \\
\hline 1 & 2.717 & 22.640 & 22.640 & 1.845 & 15.374 & 15.374 \\
\hline 2 & 1.446 & 12.050 & 34.690 & 1.719 & 14.327 & 29.701 \\
\hline 3 & 1.302 & 10.847 & 45.537 & 1.618 & 13.483 & 43.183 \\
\hline 4 & 1.168 & 9.735 & 55.272 & 1.451 & 12.089 & 55.272 \\
\hline 5 & .971 & 8.094 & 63.367 & & & \\
\hline 6 & .794 & 6.615 & 69.982 & & & \\
\hline 7 & .742 & 6.180 & 76.162 & & & \\
\hline 8 & .673 & 5.605 & 81.767 & & & \\
\hline 9 & .608 & 5.065 & 86.832 & & & \\
\hline 10 & .570 & 4.749 & 91.580 & & & \\
\hline 11 & .556 & 4.631 & 96.212 & & & \\
\hline 12 & .455 & 3.788 & 100.000 & & & \\
\hline
\end{tabular}

表4 旋转后的成分矩阵。

\begin{tabular}{lllll}
\hline 主成分 & $\mathbf{1}$ & $\mathbf{2}$ & $\mathbf{3}$ & $\mathbf{4}$ \\
\hline X12 & 0.769 & - & - & - \\
X10 & 0.738 & - & - & - \\
X11 & 0.733 & - & - & - \\
X5 & - & 0.770 & - & - \\
X6 & - & 0.737 & - & - \\
X4 & - & 0.645 & 0.791 & - \\
X1 & - & - & 0.736 & - \\
X3 & - & - & -.477 & 0.785 \\
X2 & - & - & - & 0.622 \\
X9 & - & - & - & 0.599 \\
X8 & - & - & & \\
X7 & - & & & \\
\hline
\end{tabular}

\subsection{3. 计算因子综合得分}

依据“是否进入过军队体制”变量将样本划分为两个总体, 回答为“是”的样本记为总体 1 , 回答为“否”的记为总体 2 。 两总体公因子得分情况的描述统计信息列于表 5 。通过比较看出, 总体 1 在四个公因子上的表现均好于总体 2 。

表5 因子得分的描述统计信息。

\begin{tabular}{|c|c|c|c|c|c|}
\hline 公因子 & 总体 & 最小值 & 最大值 & 均值 & 标准 偏差 \\
\hline \multirow[b]{2}{*}{ GOV } & 1 & -2.32274 & 2.74943 & .1440748 & 1.06441630 \\
\hline & 2 & -2.65450 & 1.53175 & -.1427156 & .91459771 \\
\hline LIF & 1 & -2.62822 & 2.26669 & .0782496 & 1.08287697 \\
\hline \multirow{2}{*}{ SOC } & 1 & -4.08796 & 2.39329 & .1781033 & .95712551 \\
\hline & 2 & -3.75528 & 2.27775 & -.1764231 & 1.01463938 \\
\hline \multirow{2}{*}{ POL } & 1 & -2.37309 & 2.58018 & .1809301 & .97356650 \\
\hline & 2 & -2.74213 & 2.19269 & -.1792232 & .99801436 \\
\hline
\end{tabular}

在计算出每个因子的得分情况后, 为了对各总体的制度认可情况进行综合客观评价, 本文以因子的特征根方差贡 献率为权重对各因子得分加权。综合得分函数为:

$$
\mathrm{SCO}=0.154 \times \mathrm{GOV}+0.143 \times \mathrm{LIF}+0.135 \times \mathrm{SOC}+0.121 \times \mathrm{POL}
$$




\subsection{4. 差异性分析}

上述直观对比后发现两总体在制度肯定性因子上存 在差异, 为了验证这种差异的显著程度如何, 本文采用单 因素方差分析 [14]进行探究。方差分析是基于分析数据变 异的情况从而推断两个及以上样本均数所代表的总体均
数是否存在显著差异的一种统计方法。本文将“综合得分” 作为因变量, “是否进入过军队体制”作为因子, 首先进行 方差齐性检验。从表 6 结果看, 各组方差在 0.05 的显著水 平下没有明显差异, 具有方差齐次性, 可以进行方差分析。

表6 方差齐性检验

\begin{tabular}{lllll}
\hline & 莱文统计 & 自由度 $\mathbf{1}$ & 自由度 2 & 显著性 \\
\hline 基于平均值 & 1.840 & 1 & 209 & .176 \\
基于中位数 & 1.493 & 1 & 209 & .223 \\
基于中位数并具有调整后自由度 & 1.493 & 1 & 202.679 & .223 \\
基于剪除后平均值 & 1.729 & 1 & 209 & .190 \\
\hline
\end{tabular}

方差分析结果如表 7 所示。组间均方为 1.315 , 组内均方为 0.071 , 二者之比得到F统计量的值 18.490 , 显著性小于 0.05 , 因此可以果断推翻原假设：不同水平间无明显差异，即两总体间存在显著差别。假设一得到验证。

表7 方差分析结果。

\begin{tabular}{llllll}
\hline & 平方和 & 自由度 & 均方 & F & 显著性 \\
\hline 组间 & 1.315 & 1 & 1.315 & 18.490 & .000 \\
组内 & 14.862 & 209 & .071 & & \\
总计 & 16.177 & 210 & & & \\
\hline
\end{tabular}

\section{2. 验证性因素分析}

\subsection{1. 假设模型}

基于前文提出的假设，我们可以得到图1所示的潜变 量间路径关系的概念图。

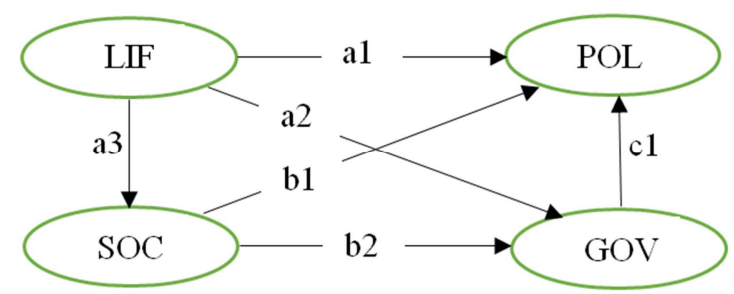

图1 制度肯定性因子的路径假设图示。

\subsection{2. 模型拟合度检验}

模型整体模式的拟合度检验旨在验证模型整体上是 否具有效度。检验模型适配度的指标有绝对适配指标、增 值适配指标和简约适配指标 [15]。本文选取表 8 中列出的 6
个指标对模型进行检验, 通过与适配度标准对比显示, 本 研究提出的模型构架与实际观察数据的拟合情况良好, 可 用以下一步的路径假设验证。

表8 模型拟合度检验。

\begin{tabular}{lll}
\hline 指标 & 模型适配度标准 & 本模型适配值 \\
\hline 卡方值 & $\mathrm{p}>0.05$ (未达显著水平) & 0.109 \\
卡方值 $/ d f$ & $<3$ & $60.345 / 48$ \\
RMSEA & $<0.05$ 优 $(<0.08$ 良) & 0.035 \\
NNFI & $>0.90$ 以上 & 0.938 \\
CFI & $>0.90$ 以上 & 0.955 \\
SRMR & $<0.05$ 优 $(<0.08$ 良) & 0.051 \\
\hline
\end{tabular}

\subsection{3. 假设检验结果 [16]}

在 $p<0.05$ 的显著水平下, $\mathrm{LIF} \rightarrow \mathrm{SOC} 、 \mathrm{SOC} \rightarrow \mathrm{GOV}$ 达到显著水平, 表明生活满意度因子对社会态度因子有显 著正向影响, 假设二得到验证; 社会态度因子对政府满意 度因子有显著正向影响, 假设三得到验证。 $\mathrm{SOC} \rightarrow \mathrm{POL}$ 在 0.1 的显著水平下接近于显著, 表明社会态度因子对政治 态度因子也存在着一定程度的影响。假设四不成立。

表9 路径系数。

\begin{tabular}{|c|c|c|c|c|c|}
\hline 路径 & Std.Err & z-value & $\mathbf{P}(>|\mathbf{z}|)$ & Std.all & 检验结果 \\
\hline $\mathrm{SOC} \rightarrow \mathrm{POL}$ (a1) & 0.139 & 1.560 & 0.109 & 0.469 & 显著 \\
\hline $\mathrm{LIF} \rightarrow \mathrm{POL}(\mathrm{b} 1)$ & 0.101 & -0.638 & 0.523 & -0.088 & 不显著 \\
\hline $\mathrm{GOV} \rightarrow \mathrm{POL}(\mathrm{c} 1)$ & 0.079 & 0.331 & 0.741 & 0.049 & 不显著 \\
\hline $\mathrm{SOC} \rightarrow \mathrm{GOV}$ (a2) & 0.145 & 3.112 & 0.002 & 0.526 & 显著 \\
\hline $\mathrm{LIF} \rightarrow \mathrm{GOV}(\mathrm{b} 2)$ & 0.186 & 0.724 & 0.469 & 0.100 & 不显著 \\
\hline $\mathrm{LIF} \rightarrow \mathrm{SOC}(\mathrm{a} 3)$ & 0.240 & 3.037 & 0.002 & 0.463 & 显著 \\
\hline $\mathrm{a} 2 \mathrm{c} 1$ & 0.035 & 0.340 & 0.734 & 0.026 & 不显著 \\
\hline $\mathrm{b} 2 \mathrm{c} 1$ & 0.013 & 0.282 & 0.778 & 0.005 & 不显著 \\
\hline total & 0.276 & 5.455 & 0.000 & 1.548 & 显著 \\
\hline
\end{tabular}


依据上述检验结果, 将假设模型中不显著的路径删除, 得到图2中修正后的路径图。

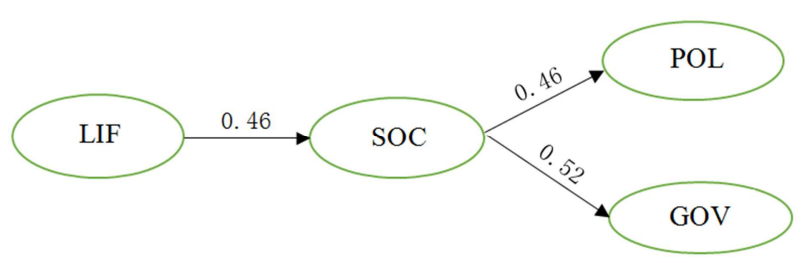

图2 修正后的路径图。

\section{4. 结论}

当下, 我国正面临着全方位的挑战和更加复杂的外部 环境, 传统安全威胁与非传统安全交融, 各类风险不断累 积, 为确保在实现现代化进程中的平稳有序, 内部环境安 全稳定至关重要。经过学者们的长期探索, 发现安全形势 呈现出“国家安全的根本在于政治安全, 政治安全的核心 在于制度安全”这样一个现实逻辑, 而国民对我国现有制 度的肯定又是制度安全的重要体现。

为切实衡量当下国民的制度肯定性因子水平, 本文从 有着较高政治意识要求的军人群体出发, 基于生活满意度、 社会态度、政府满意度以及政治态度四个层面从CGSS (2015) 问卷中抽取相关的十二个问题, 对军队体制内外 的制度认可情况作以对比, 并提出四个假设。通过结构方 程模型的验证, 结果表明体制内人员和体制外人员在对现 有制度的认可程度上是存在显著差异的, 且生活满意度会 影响一个人的社会态度, 社会态度又会正向作用于政治态 度和政府满意度, 即假设一至假设三成立。因此, 将军队 的思想武器武装到每一个国民，实现意识形态领域的“全 民皆兵”这一设想得到了初步的支撑。将着眼点放在提升 公民生活幸福感、增强公民社会信任程度、提高政府工作 效果及效率上, 将会有效引导公民政治态度的正向转变, 进而对我国的制度安全产生积极作用以及有力维护。

\section{参考文献}

[1] 武贤明.国家安全思想的哲理内涵论析[J].长春工业大学学 报(社会科学版),2008 (02): 38-41。
[2] 李家恒.国家安全的构成要素论析-谈学者与习近平主席的 细微认知差异 [J].四川警察学院学报,2015,27(02):128-134。

[3] 马振超.当代中国政治安全的现实逻辑和终极价值[J]. 国际 安全研究,2018, 36 (03): 3-22+156。

[4] 张星. 政治安全的制度之维[D].武汉大学,2013。

[5] 潘晓珍. 理论、制度与现实: 全球治理时代中国国家能力建 设的三维审视[D].苏州大学,2015。

[6] 任海平,王天龙.当前我国国家安全形势综合评估及应对 [J]. 全球化,2015 (01): 61-71+132。

[7] 王芙蓉,张海明,朱雪玲.军人忠诚培育研究综述[J].中国临床 心理学杂志, 2019,27 (03): 628-633+643。

[8] 王龙飞. “全民皆兵”的缔造:抗战时期太行根据地的军区体 制 [J].抗日战争研究,2017 (04): 17-32。

[9] 市玉梅. 结构方程模型研究及其应用 [D]. 大连海事大 学, 2017。

[10] 沐守宽,顾海根.探索性因素分析因子抽取方法的比较 [J].心 理学探新,2011,31 (05): 477-480。

[11] 何霞,刘文明.高职院校教师激励模型的验证性因素分析-基 于28所高职院校的抽样调查 [J].中国高教研究,2014 (03): 95-100。

[12] 解素雯. 基于主成分分析与因子分析数学模型的应用研究 [D].山东理工大学,2016。

[13] 王春枝.因子分析中公因子提取方法的比较与选择 [J].内蒙 古财经大学学报,2014, 12 (01): 90-94。

[14] 牛凯.数据分析之单因素方差分析[J].产业与科技论坛,2019, 18 (02): 57-58。

[15] 兰国帅,钟秋菊,吕彩杰,宋亚婷,魏家财.探究社区量表中文 版的编制-基于探索性和验证性因素分析 [J].开放教育研 究,2018, 24 (03): 68-76。

[16] 张彦君. 粮食直接补贴政策效果及影响路径分析 [D].西北 农林科技大学, 2017。 